\title{
WILL ELECTRIC DRIVERLESS CARS KILL BUS AND LIGHT TRAIN OPERATIONS?
}

\author{
SERGE VAN THEMSCHE \\ Consultant at PM\&BD Consulting UG, Berlin, Germany.
}

\begin{abstract}
Private transport is associated with conventional cars. Cars pollute, run empty during peak hours, stay idle $95 \%$ of the time and are privately owned. Fifteen years from now, the first driverless cars will start hitting the road in large numbers. By then most cars will be electric. If the service-oriented business model takes on momentum, a fleet of unmanned taxis will compete directly with buses and light trains. At that point in time, differences between public and private transport will only be semantic. After all, metro, buses and cars will all be unmanned, electric and managed by a fleet operator. As a consequence, city developers will have problems, justifying subsidies to bus and light vehicle operations. Without these subsidies, buses and light trains will have problems, competing with car transport. Unmanned taxis will cut journey cost by around $60 \%$ to $80 \%$. New taxi apps allowing for car sharing will slash cost even further, making unmanned taxi fares unbeatable. Furthermore, driverless taxis will provide better services, picking up and dropping off passengers where they want to rather than stopping at each station. So if unmanned taxis are cheaper and provide better service, what selling arguments are left for buses and light metros? Transport capacity? Not really! In fact, a fleet of unmanned taxis using platooning technology (cars following each other at one-metre distance) and car sharing apps would achieve around 15.000 PPHPD per lane, more than a bus and equal to light train operations.

Keywords: BRTs, carpooling, driverless technologies, electric cars, energy consumption, environment, hailing taxi apps, platooning, transport capacity, unmanned cars.
\end{abstract}

\section{DIFFERENCES BETWEEN PUBLIC AND PRIVATE TRANSPORT}

If we were to ask people, in which transport categories metros, buses or cars would fit in, we would obtain an easy and quick answer. When asked if taxis would be considered public or private transport, they probably would hesitate. Some would consider taxis as public and would allow them to ride on the dedicated lanes together with buses, while others would force them to ride with ordinary traffic. The fact is that taxis don't tick in the boxes of the four concepts that usually set the boundaries between public and private transportation: environment, driving, ownership and social role. What we will now demonstrate is that new car technologies will make these distinctions completely outdated.

\section{ENVIRONMENT}

Currently public transport is considered environmentally friendly, while cars are viewed as the main villains. Three fundamental concepts are underlining this view: energy consumption, pollution emission and dedicated space allocation. 


\subsection{Energy consumption}

Electrical cars have started gaining momentum. Several new technologies or manufacturing techniques have been able to improve the performance of electric vehicles, making them more attractive. Substitution of steel products by lighter materials such as plastic or aluminium has reduced the energy required to move on the vehicle mass. Battery performance has been doubling every three years for the last two decades, thanks to the use of new material and new mass production techniques. Changes in power outlet capacities, allowing for higher current and voltage, as well as DC charging, are expected to reduce charging time from the current 4 to 5 hours to somewhere in the range of 5 to $10 \mathrm{~min}$, a time lapse which in our view will make it possible to find recharging facilities along the roads, happy to sell electricity at a profit. Furthermore, new smart charging connectors will allow the use of electric cars to play a storage role, one of the key limiting factors today of renewable energies. In other words, electric cars will be seen as an enabler of new carbon-free technologies.

The second negative view about cars is that they consume more energy per passenger than buses or trains. This is partially true. Cars are much lighter on $\mathrm{a}^{2}$ basis (i.e. on average, two and three times lighter than buses and trains respectively). As mass is one of the main energy consumption for inner city operations (i.e. mainly from accelerating and decelerating), cars are theoretically more energy efficient per kilogram transported. Moreover, electric vehicles can also recuperate kinetic energy obtained while braking and may even have no drive train, thus improving even further their overall energy efficiency. With all these features, electric vehicles are around four times more efficient than conventional cars in terms of energy consumption 'wheel-to-wheel'.

When we take into consideration energy production inefficiencies for fossil fuel (i.e. during drilling and fuel transport) and electricity (i.e. losses during generation, distribution and charging), electric cars are still more energy efficient. For countries such as America, France and Brazil this so-called energy consumption 'wall-to-wheel' can represent better efficiencies in the range of $70 \%, 80 \%$ and $200 \%$, respectively, when taking into account these countries' generation matrix. In our book [1], we've compared energy consumption between cars, buses and trains. In order to do this and be fair, we needed to introduce the notion of occupancy. A train during peak hour can transport four passengers $/ \mathrm{m}^{2}$ (even six to eight in the poor megacities of this world), while cars transport in Europe and North America only 1.1. When many inefficiencies are factored in, we do see that electric cars aren't as energy efficient as trains when comparing wall-to-wheel but still do better than diesel buses (Table 1).

Table 1: Adaptation from French Environment Agency ADEME* and US BTU information** including occupancy rate on inner city trips. Source: Author [1].

\begin{tabular}{lcccc}
\hline Transport mode & \multicolumn{4}{c}{ Real KWh per passenger km } \\
& Wheel-to-wheel & Wall-to-wheel & Wheel-to-wheel & Wall-to-wheel \\
\hline $\begin{array}{l}\text { Car petroleum } \\
\text { based }\end{array}$ & 0.78 & 0.88 & 1.66 & 2.00 \\
Electric car & 0.24 & 0.58 & 0.51 & 1.32 \\
Bus diesel based & 0.39 & 0.44 & 1.66 & 2.00 \\
Rail & 0.11 & 0.19 & 0.71 & 1.19 \\
\hline
\end{tabular}


However, if we were to consider cars running at full capacity, carrying on four to five passengers due to the new service business models we will describe further on rather than the usual 1.1 during peak hours, we do see that electric car's energy consumption values are totally comparable to commuter trains.

\subsection{Pollution emission}

There are two principles that determine how much pollution generated by transport contribute negatively to health issues.

- Because people live mainly in cities, where pollutants are emitted is more important than how much are emitted.

- The more a vehicle consumes energy, the more likely it is to emit pollutants, as pollution is directly linked to motor inefficiencies.

The point is that in the future, when all cars will become electric, there will be almost no differences from a pollution perspective between the three transport modes on these two principles. Moreover, whenever electric cars will be connected to smart grids, it will be possible to use the cars' battery to store carbon-free energy. By storing energy at such times when wind stops blowing and night falls, electric cars could even boost investment in renewable energy.

\subsection{Dedicated space allocation}

A service model of unmanned cars will reduce the number of cars that stay idle on the side of the roads. This will free space reserved for parking areas, allowing city planners to either increase occupation density or build more green parks.

\section{DRIVERLESS CARS}

Before unmanned cars start hitting the roads, a continuum of new technologies will be introduced to the market, bringing increasing driving support functions (e.g. change of lane, upfront braking info, etc.) and new automating functions (e.g. automated parking, automatic emergency calls, etc.). These new technologies won't change fundamentally the concept of who's in control of the vehicle. The person behind the wheel or holding the car will continue to be responsible for accidents and fines. However, whenever cars become completely driverless, a combination of onboard and wayside technologies will be managing solely the car. This technological revolution, which is already making drivers redundant in the railway industry, will also happen in the automotive and bus industries. As most of the accidents are caused by human errors, no forces will go against a technology that will slash the number of deaths and injuries.

\section{VEHICLE OWNERSHIP MODEL}

In the past, public transport companies owned and operated their own fleet of buses, metros, commuters or trains. Nowadays private or public companies must increasingly compete to obtain the right to operate concessions. While private companies obviously are there for the profit, national champions such as SNCF, RATP or Deutschbahn have had to adapt their mindset to the same economical rules. To become competitive, they often must team up with financing companies called ROSCOs, which buy and lease fleets, like the private companies do. Thus, fleet ownership is now more a consequence of the private -public partnership rules than an absolute necessity. 
The automotive industry - until recently - hadn't really been confronted with such disrupting trends. Obviously car rental companies or car fleet operators have been around, but this service model was more the exception than the rule. However, lately innovative business models have emerged, enabled by new Internet apps, reducing car ownership appeal. They can be regrouped under three different categories: rental by the hour, car sharing and carpooling. Inner city residents, usually young, are attracted to rental by the hour (e.g. from companies such as Car2Go) or car sharing and pooling services because they don't see the point in investing in equipment that stays idle most of the time and costs so much to park and operate. For the younger generation, surveys have also shown that the social status that their parents or grandparents associated with the possession of a nice car is long gone. These services competing with public transport are fast becoming trendy not only among the pioneering fringe of the city population (e.g. Blablacar is popular even among senior citizens). However, the biggest threat to the car ownership model will come from taxi-like services. Everyone has heard about Uber and the strong reactions this company has generated within the taxi community, throughout the world. In just a few years, Uber and its competing hailing firms have stolen market shares to normal taxi operators. They have been extremely popular not only because they are cheaper than conventional taxis, but also because they are convenient and reliable. Moreover, the services they provide ensure traceability and responsibility through their hailing apps, in an industry well known for freewheeling. These Internet hailing apps have allowed city residents to use road transport without owning a car. Now imagine how attractive such a hailing service would become if only fares were similar to that of a bus or metro ticket.

\section{SOCIAL ROLE}

Public transport plays a crucial social role, enabling low-revenue citizens to go to work and students to attend school without having to invest in expensive equipment. It is also enabling an increasing elderly population to stay in their own home, delaying as long as possible the moment when they need to leave for a retirement home. In order to play this social role, governments accept to subsidize public transport. As many public transport users come from the poorest social class, these subsidies are perceived as socially fair measures. On the other hand, car users are perceived as rich individuals who can afford to pay excise taxes on their vehicle and gasoline purchases, as well as hefty parking costs. Cross subsidies are put in place to encourage car users to switch to public transport.

Carpooling or sharing as well as rental by the hour is changing the negative perception of city planners towards users of these car services. Special parking rights are allocated for rental by hour services and pooling facilities are developed along many big arteries. In many cities, taxis have also been perceived as playing a social role as taxis must accept all clients, regardless of where they want to go, or if they have a physical handicap requiring some kind of support. Cheaper than ambulances, they are even used to replace them to transport mobile patients to and fro treatment facilities. As such, they have been granted in many cities the right to ride on dedicated bus lanes.

\section{UNMANNED ELECTRIC HAILING TAXI SERVICES}

Car speed within inner city boundaries is increasingly restricted to a maximum speed limit of $50 \mathrm{~km} / \mathrm{h}$. However, average city car speed, especially during peak hours, is usually nearer $20 \mathrm{~km} / \mathrm{h}$. This makes it extremely attractive to start the deployment of unmanned technologies for such an operational environment. Indeed, for operations under $50 \mathrm{~km} / \mathrm{h}$, the legal 
responsibilities will be much less severe. This is probably why Uber has already announced that they wish to develop driverless technologies or Google (which is already running unmanned car technology for many years) invested in such company.

An electric unmanned hailing taxi operation would tick all the boxes of a public transport service. It would be environmentally friendly, as it would be electric. It wouldn't be privately driven or owned. Being driverless and managed by a fleet operator it would also qualify as public transport.

As seen, taxis are already perceived as playing a social role, though still considered expensive. Studies have shown that unmanned taxis will slash the fare of an inner city trip by around $80 \%$. This means that unmanned taxis' fare for such trips will be at par with metro or bus tickets. However, Uber came up already with apps that allow taxi-pooling services. If unmanned cars were to provide these same pooling services for four clients simultaneously, this already reduced fare could even be shared between these clients. Such pooling services would be unbeatable price-wise but also service-wise. Indeed, taxis are already more comfortable than buses or metros. Unlike buses and metros, that provide point-to-point operations (i.e. to go to station D from station A, they need to stop at B and C), such pooling taxi service could provide multi-point-to-multi-point operations (i.e. from any point $\mathrm{A}$ to any point $\mathrm{D}$, without having to stop at stations $\mathrm{B}$ and C). In other words, unmanned electric hailing/ pooling taxi services would offer a better public service for much less. In indebted countries, reducing or eliminating transport subsidies altogether will be an extremely appealing argument that will surely influence transport investment decisions.

If such taxi services are better, cheaper and tick all the public transport boxes, which competitive advantage will train and bus technology still have? Higher capacity is surely the remaining advantage. Well, we will now see that this won't be the case for medium capacity transport mode.

\section{TRANSPORT CAPACITY}

Comparing transport capacity of the various transport modes isn't easy. Though train, bus and car's capacity obey to the same four variables, the way they are impacted can differ tremendously. These four variables are the vehicle's own capacity, number of vehicles running on the system, average speed and headway.

\subsection{Vehicle capacity}

Vehicle capacity is defined by the number of passengers that can be transported. Four elements will have an influence: physical characteristics, safety standard, comfort level and occupancy rate.

The vehicle's physical characteristics, especially its dimension, will have a huge impact on capacity. Vehicles can be built larger, longer or even with double-decks. Manufacturers can also improve their design (e.g. by putting equipment on the train's roof) to increase capacity. The network's physical limitations will directly influence the vehicle's shape. Station clearance and length, tunnel envelope calculated with jerking, type of electric grid connection, etc., will influence directly the trains' capacity. Roads on the other hand are more standardized, which explains why cars and buses' width don't vary much (though maximum bus length can be restricted by tight curves). Safety and operational standards (e.g. places allocated to physically handicapped or obese people) will also affect standees and seated passenger places. Transport Authorities might as well decide to change the comfort level by, for 
instance, reducing number of seats and inner space dedicated to seating area. More drastic attitude can be considered such as tolerating a higher volume of standees per area, affecting directly the level of passenger comfort. In most countries, this level is four passengers $/ \mathrm{m}^{2}$, but in some megacities of South America and India, they apply eight passengers $/ \mathrm{m}^{2}$ for constructive requirements. This measure almost doubles the capacity instantly, but at the detriment of the quality of ride.

\subsection{Number of vehicles}

There is a maximum number of vehicles that can run on a track or lane, at any given time. However, till this number is reached there is always a possibility to add extra capacity. Once this number is reached, there is no point in adding more vehicles, as the extra vehicles would only stay idle or would need to slow down to maintain the same safety spacing.

\subsection{Average speed}

The speed of a line or lane is function of the inherent characteristic of the vehicle, the safety and comfort of passengers, as well as the limiting factors of the network operation. For instance, vehicle's acceleration, maximum speed and deceleration are key factors influencing the overall system speed. In most metro applications, because distances between two stations are short, the trains are almost always accelerating and decelerating. Metro car motorization is usually adapted for those specific conditions. However, metro operators must also take into consideration the safety and comfort of the passengers when accelerating and decelerating. After all, they don't want the passengers to fly through the windshield, when applying the brakes. Another limiting factor to speed is the network operation. Average speed clearly changes according to the type of railway application (e.g. metro average speed: $35 \mathrm{~km} / \mathrm{h}$ ).

Although more extreme car acceleration and speed possibilities are usually more related to the driver's wealth and his willingness to show it off, in most countries even the cheapest automobiles aren't limited by their inherent motorization characteristics. Infrastructure specificities, weather conditions or legal speed limitation are the main speed restricting factors. Traffic light and potentially any pedestrians, bicycles, cars or objects blocking the road access will also influence speed. Bus speed will be influenced by a mixture of all this, which explains why, in general, buses running in urban areas can't drive over $20 \mathrm{~km} / \mathrm{h}$.

\subsection{Headway and interspacing}

The distance between two vehicles moving in the same direction is important for network capacity because it limits the number of vehicles that can be added to this system without reducing the overall speed. The terms 'interspacing' and 'headway' are usually used for cars/ buses and trains, respectively. In both cases, distances are transformed into time. For cars, people maintain naturally the equivalent of a 2-second minimum distance, regardless of their speed. The railway industry has developed new electronics and powerful software, reducing significantly the headway without increasing safety risks. The evolution of signalling technologies from fixed blocks to moving block have reduced headways from the $120-150$ s range to $60-90 \mathrm{~s}$, doubling almost instantaneously the throughput capacity of a metro network. 


\section{TRANSPORT MODE CAPACITY COMPARISON}

Comparing different transport modes isn't easy because they include different concepts. However, one capacity indicator called PPHPD (passenger per hour and per direction) can be tweaked to do so. Though operational factors, such as headway, comfort level or occupancy rate, may impact the comparison, we will select reasonable values that will, nevertheless, give an idea of how conventional and driverless cars are compared to more traditional 'public' transport mode.

\subsection{Mass transit capacity}

Metro or commuter network capacity can vary substantially from one system to another. Trains and metros come in various shapes, size, height, length and width. Station length, platform size and height, tunnel diameter and other network characteristics are also very different from one city to the other. Operational factors such as headway or frequency are all variables that affect the mass transit's capacity. The values selected in the next table are at a headway of $120 \mathrm{~s}$ reachable with conventional fixed block technology and $90 \mathrm{~s}$, mainly reachable with driverless metro technology. We've used six passengers $/ \mathrm{m}^{2}$, a comfort level acceptable in developing countries. We should also notice that the number of heavy metro cars could be extended to 12 cars, doubling this capacity (Table 2).

\subsection{Bus and Bus Rapid Transit (BRT) capacity}

Bus capacity on conventional lines is more standardized. The following table gives a direct comparison, using a six passengers $/ \mathrm{m}^{2}$ comfort level (Table 3).

Since its first introduction in Brazil 40 years ago, operational concepts have been improved and have transformed BRTs into a direct competitor of medium-sized metro systems. Buses depart the lane and go to curbside spaces where they can stop to unload and load passengers. There can be up to five such loading spaces, called berths, per bus stop. Dwell time is thus not on the critical pass for overall BRT capacity, as load can be done in parallel. In other words,

Table 2: Commuter train capacity. Source: Author [1].

\begin{tabular}{llccrc}
\hline Technology & \multicolumn{1}{c}{$\begin{array}{c}\text { Description } \\
6 \text { passengers } / \mathrm{m}^{2}\end{array}$} & $\begin{array}{c}\text { No of cars } \\
\text { No }\end{array}$ & $\begin{array}{c}\text { Capacity } \\
\text { Unit }\end{array}$ & $\begin{array}{c}120 \mathrm{~s} \\
\text { PPHPD }\end{array}$ & $\begin{array}{c}\text { PPHPD } \\
\text { PPH }\end{array}$ \\
\hline Heavy metro & Small body $(\mathrm{Rc}+$ & 6 & 1016 & 30500 & 40600 \\
& M $+\mathrm{M}) \times 2$ & 6 & 1508 & 2500 & 60300 \\
& Medium body ( & 6 & 1736 & 2100 & 69400 \\
& Rc $+\mathrm{M}+\mathrm{M}) \times 2$ & & & & \\
& Large body ( Rc + & & & & \\
& M $+\mathrm{M}) \times 2$ & 7 & 1002 & 30080 & 40000 \\
Monorail & 7 -car train & 1 & 270 & 8100 & 10800 \\
Tramway & $30 \mathrm{~m}$ & 2 & 540 & 16200 & 21700 \\
& $30 \mathrm{~m} \times 2($ cou- & & & & \\
\hline
\end{tabular}


Table 3: Bus capacity. Source: Author [1].

\begin{tabular}{lccccc}
\hline Technology & $\begin{array}{c}\text { Description } \\
6 \text { passengers } \\
/ \mathrm{m}^{2}\end{array}$ & $\begin{array}{c}\text { No of cars } \\
\text { No }\end{array}$ & $\begin{array}{c}\text { Capacity } \\
\text { Unit }\end{array}$ & $\begin{array}{c}120 \mathrm{~s} \\
\text { PPHPD }\end{array}$ & $\begin{array}{c}90 \mathrm{~s} \\
\text { PPHPD }\end{array}$ \\
\hline $\begin{array}{l}\text { Standard bus } \\
\text { type, } 1 \text { lane, } 1\end{array}$ & $\begin{array}{c}\text { Articulated } \\
\text { stopping bay, } \\
\text { onboard }\end{array}$ & 1 & 85 & 2250 & 3400 \\
\hline
\end{tabular}

and if we didn't take into consideration the departing and reinjection into the lane, all five buses could take only $20 \mathrm{~s}$ dwell time, if all these buses were synchronized perfectly (this is impossible). Having said that, a BRT such as the Transmilenio in Bogota (Table 4) can carry as much as 35.000 PPHPD!

\subsection{Road throughput}

For conventional cars running on one highway lane, car throughput (i.e. number of cars per road section) is limited to around 1.800 cars. This number isn't proportional to the number of lanes, as vehicles changing lane, the number of heavy vehicles, lane width and driving habits would affect this throughput. For standard roads, throughput is a little more complicated to calculate as street lights, stops, or possibilities to turn at junctions, as well as potential interference such as pedestrian and bicycle lanes, would also influence. Intelligent lighting systems, integrating video cameras on an IP network, could improve the number, by reducing waiting time. Without considering driverless technologies, the maximum highway lane capacity could be easily calculated (i.e. throughput $\mathrm{x}$ occupancy rate of five passengers per car) at 9.000 PPHPD. However, we all know that such a number grossly overestimates the real capacity. In most European and North American countries, peak hour and average occupancy rates are 1.1 and 1.5 , reducing the highway lane capacity to 2.000 PPHPD and 2.700 PPHPD, respectively. One interesting aspect of new e-mobility technologies is the possibility to do platooning (i.e. convoying of cars). Under a main vehicle leadership, cars follow each other a few metres apart, thanks to vehicle to vehicle (V2V) communication. Adopting such driving technologies will increase highway throughput. How much will depend on whether cars adopt the safety principle called 'brick wall' (i.e. a vehicle must mandatorily stop prior to the absolute position of the vehicle) or not.

Table 4: BRT capacity. Source: Author [1].

\begin{tabular}{lc}
\hline Location & PPHPD \\
\hline Bogota, Colombia & 35000 \\
Guangzhou, China & 26900 \\
Curitiba, Brazil & $13900-21000$ \\
Istanbul, Iran & $7300-19500$ \\
New Jersey, USA & 15500 \\
\hline
\end{tabular}




\subsection{Road throughput with V2V capability}

Platooning technology may consider two weaker safety criteria than the 'brick wall' principle.

- If a vehicle applies maximum braking until it comes to a stop, the following vehicle should be able to stop without colliding into it, if the interspacing between the two cars is sufficient. Such a hard braking disturbance may arise on an automated highway system in response to an obstacle or a vehicle malfunction.

- If a vehicle applies maximum braking and the following vehicle collides with it, the relative velocity at initial impact should be small. People maintain the same safety distance of around $2 \mathrm{~s}$ and thus tend to use the same speed. The low relative velocity safety criterion can be met if the vehicles are either far apart or close enough to one another. In the former case the vehicles have time to stop before they collide while in the latter they collide very quickly and hence the relative velocity at impact is small.

A white paper [2] 'Capacity Analysis of Traffic Flow over a Single-Lane Automated Highway System', analyses the impact of self-driving vehicles on road capacity. It indicates that if we systematically apply to the leading car, a maximum braking capacity factor lower than the worst car in the platoon, there will be no intra-collision. The goal of the intra-platoon control law considered above is to maintain constant interspacing between adjacent followers.

The study took into consideration three levels of cooperation between individual vehicles (including cars, buses and trucks), as well as a high level of cooperation between cars within a platoon. Vehicles with no communication capability or with very limited cooperation (i.e. communicating only during manoeuvres and emergencies such as hard braking) showed no capacity increase. Vehicles continuously exchanging state information (e.g. speed and acceleration), in addition to manoeuvre coordination messages and emergency warnings that used constant safety spacing, showed a slight capacity increase of around $10 \%$.

Instead of maintaining constant safety spacing, driverless cars may maintain specific adapted interspacing. Through V2V, cars can know the braking capacity and distance bumper to bumper of the preceding cars and compare it to its own braking capacity required, at any given speed. It can thus adapt the inter-vehicle spacing values to all paired vehicles on the highway, following the vehicle ahead at a safe separation distance for its actual braking capability, rather than the minimum of the distribution. Non-uniform spacing design could thus increase further the automated highway capacity, by an estimated $30 \%$.

On a highway with platooning capabilities, vehicles could travel in closely spaced groups of up to 20 vehicles. The cars within the platoon through V2V would be able to know the braking action of the other cars and maintain an intra-platoon separation of a few metres. Platoons would need to be isolated from each other by larger distances, in order to avoid inter-platoon collisions. The same study has shown that platooning could double road capacity. The principle is very simple: all cars are running at the same speed, thus avoiding any additional safety distance that drivers instinctively maintain for the 2 sheadway. In other words, all 20 cars within the platoon can maintain the same safety distance (i.e. $10 \mathrm{~m}$ ). The inter-vehicle spacing for every vehicle pair is picked using the lowest and highest values of the distribution to obtain safety in the hard braking sense. 


\subsection{Highway and road capacity}

Figure 1 gives a view based on speed and capacity of most Western countries.

The issue with this figure is that we cannot use the maximum capacity for cars expressed in PPHPD, as for buses and trains. The reason is that we know that cars run mostly empty. This is why we prefer to use an average occupancy rate of 1.5 passengers for the USA and Europe. This means that a highway theoretical capacity of 9.000 PPHPD for one highway lane with five passengers per car is reduced to around 2.800 PPHPD, at the speed of around $25 \mathrm{~km} / \mathrm{h}$.

The next figure shows the potential impact of platooning and adding an additional lane. Such a solution could help solve transit issues in gridlock urban areas, generating a capacity of 11.000 PPHPD at around $25 \mathrm{~km} / \mathrm{h}$ (Fig. 2).

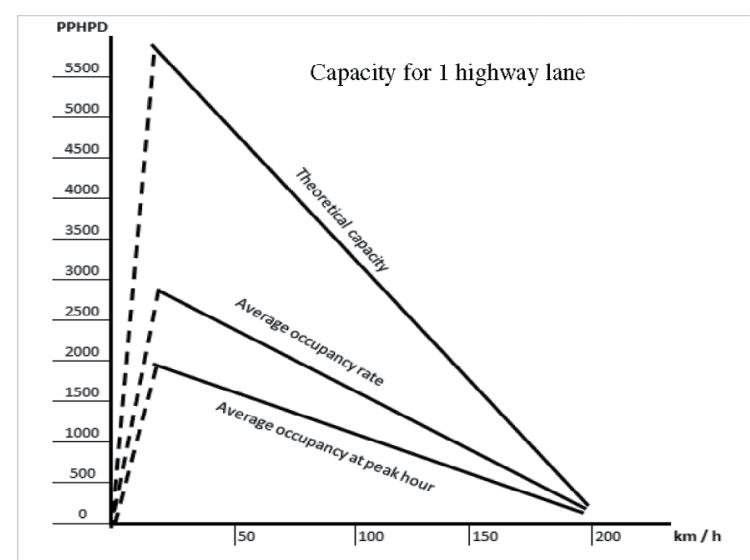

Figure 1: Capacity in PPHPD for one highway lane. Source: Author [1].

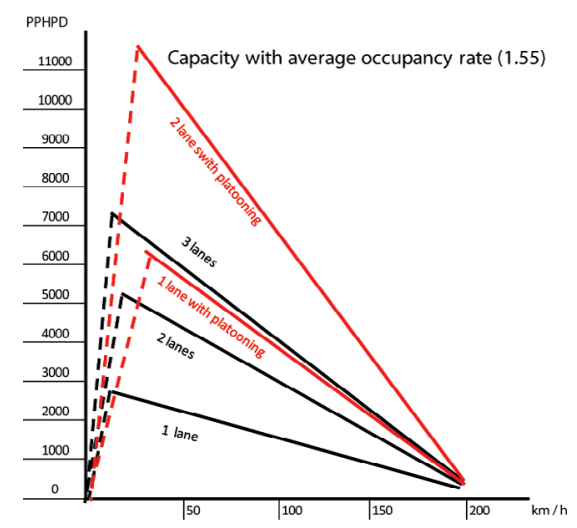

Figure 2: Capacity in PPHPD for one and two highway lanes with and without platooning services. Source: Author [1]. 


\subsection{Combining carpooling and platooning}

What the above figure doesn't show is the same platooning services on a two-lane highway, dedicated solely to unmanned taxis, operating at full capacity (i.e. four to five travellers per car) and using pooling apps. In this case, the road capacity could reach 20.000 to 30.000 PPHPD depending on operational speed or the equivalent of a medium-sized metro line and double the capacity of a tramway or LRV! Obviously these numbers would need to be tested but still show the tremendous opportunities that unmanned operations can unleash.

\section{CONCLUSION}

Unmanned cars will disrupt road transportation as we know it, making the distinction between public and private transport irrelevant, as cars will be electric, driverless and environmentally friendly. Furthermore, a fleet of driverless taxis using hailing and pooling apps will offer unbeatable price and attractive services, playing a useful social role without the need for subsidies. Capacity will still remain an issue in the big cities and will ensure that heavy metro and commuter trains continue playing a key role in city decongestion solutions. However, these same hailing and pooling taxi services running on dedicated lanes with platooning technologies could be an alternative to buses and light rail vehicles, proposing capacity in the 10.000 to 30.000 PPHPD range, depending on the number of lanes. Medium city authorities when making their transportation plan will need to take into consideration these game changer technologies.

\section{REFERENCES}

[1] Van Themsche, Serge., 'The advent of unmanned electric vehicles; The choices between E-mobility and Immobility', Springer, 2015.

[2] Michael, James B., Godbole, Datta N., Lygeros, John \& Sengupta, Raja., 'Capacity Analysis of Traffic Flow over a Single-Lane Automated Highway System' from the California PATH, Institute of Transportation Studies. 